\title{
Myringoplasty in Children: When and How. Retrospective Analysis of 15 Years Experience and Prospective Considerations
}

\author{
Enrico Maria Amadei M.D*, Beatrice Enrica Fabiano M.D., Claudio Cola M.D. \\ Department of Otorhinolaryngology and Audiology, Infermi Hospital, Rimini, Italy \\ Email: enricomaria.amadei@libero.it
}

\begin{abstract}
Myringoplasty is a safe and relatively rapid procedure to close uncomplicated tympanic perforations. Good surgical and functional results are usually expected. In Literature we can find a wider range of success rate of miringoplasty performed in children than in adults, varying from 35 to $94 \%$. We report a retrospective analysis of 15 years experience about 63 cases of type I tympanoplasty performed in children for simple tympanic membrane perforation. In our series of 63 cases, we had a success closure rate of $59 / 63$ cases (94\%) with a medium follow-up of 36 months. The Air Bone Gap closure was always found from good to very good. The aim of this study is to report the retrospective analysis of our experience about pediatric myringoplasty for simple tympanic membrane perforation, comparing these data with those published in the Literature.
\end{abstract}

Keywords: Pediatric myringoplasty; tympanic perforation; type I tympanoplasty.

\section{Introduction}

Performing a myringoplasty with success, it means to have an intact tympanic membrane with normal middle ear mucosa. As consequences we will have a reduction of infection rate, a restoration of a normal auditory function and the freedom to let water gets into a healthy ear.

The importance of this kind of surgery was also underlined by Tos and Lau [1]: they wrote that myringoplasty helps to lessen progression of ossicular pathology.

After a review of the Literature, we felt a sort of necessity to define some topics around pediatric myringoplasty, in the light of a long clinical and surgical practice. Outcomes in pediatric otologic surgery are not so omogeneous and myringoplasties performed in a pediatric population are thought to be less successful than those performed in adults [2-3].

Many studies treat pediatric ear surgery in toto, while in this revision paper we would like to focus the attention selectively on myringoplasty performed for simple tympanic membrane perforation. Inclusion criteria for this study were: defined and dry membrane perforation with normal middle ear mucosa; complete integrity of the ossicular chain and a normal contralateral function of the Eustachian tube. This information was obtained by accurate anamnesis, audiometric tests and endoscopic evaluation of the nasopharyngeal cavity. No post-traumatic perforation was considered.

After a correct patients selection and accurate pre-operating evaluations we can show the results of 63 myringoplasties performed in 5 to 16 aged patients and we discuss our experience comparing to those present in the Literature.

\section{Material and Methods}

We submitted for type I tympanoplasty (myringoplasty) 63 patients aged between 5-16 years old in the period 2000-2015. We performed a minimum follow-up of 18 months, with mean period of surveillance of 36 months. They were 36 females and 27 males.

We usually wait at least 8 months, before performing this kind of surgery. During this time the patient has been informed to avoid water entrance in the ear, to avoid strong blowing of the nose and to clean regularly nasal cavities.

We found a central perforation in 55/ 63 cases (87\%); a peripheral one in 8/63 cases (13\%).

The exclusion criteria are: retraction pockets, cholesteatoma, tympanosclerosis, revision surgery, ossicular chain disorders. We paid particular attention to the anatomical features of the contralateral ear. 
About surgical technique we performed everytime an underlay myringoplasty, preceded by a routine canalplasty and a retroauricular approach in every patient in general anesthesia, with the exception of 4 cases. In particular, these 4 patients were the older of our cases (14, 14, 15 and 16 years old), and the tympanic membrane perforations were small and posterior: in these 4 cases we used local anesthesia and a transcanalar approach.

Graft materials consist in temporalis fascia and tragal cartilage with pericondrium. No allogenic tissues were used.

We performed a cartilaginous reconstruction with a palisade technique in the first 10 patients, using thin pieces of tragal cartilage to support the temporalis fascia. Then after unsatisfactory results, we changed our technique. So we started to place a single larger cartilaginous disk plus pericondrium, with the same size as the calibrated external auditory canal, applied using the underlay technique below the temporalis fascia and the previously elevated tympano-meatal flap [Fig 1-4].

All the ears were packed with gelfoam and the retroauricular incision was closed with sorbable intradermic suture.

All the patients underwent to preoperative tonal audiometry and contralateral impedenzometry to study bilateral function of Eustachian tube. Postoperatively the exams were performed 1 month and 6 months later.

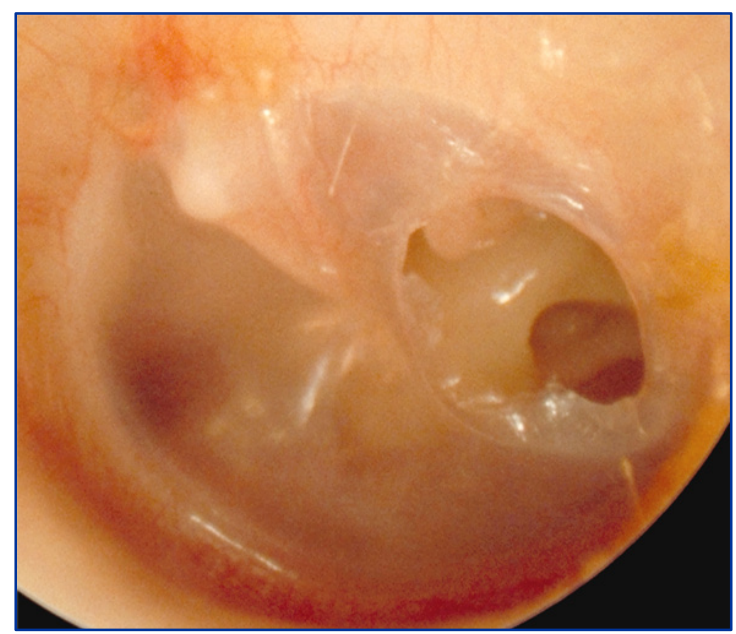

Figure 1. Left Ear. Perforation of the posterior part of the left eardrum, with healthy middle ear mucosa and without otorrhea or ossicular erosion.

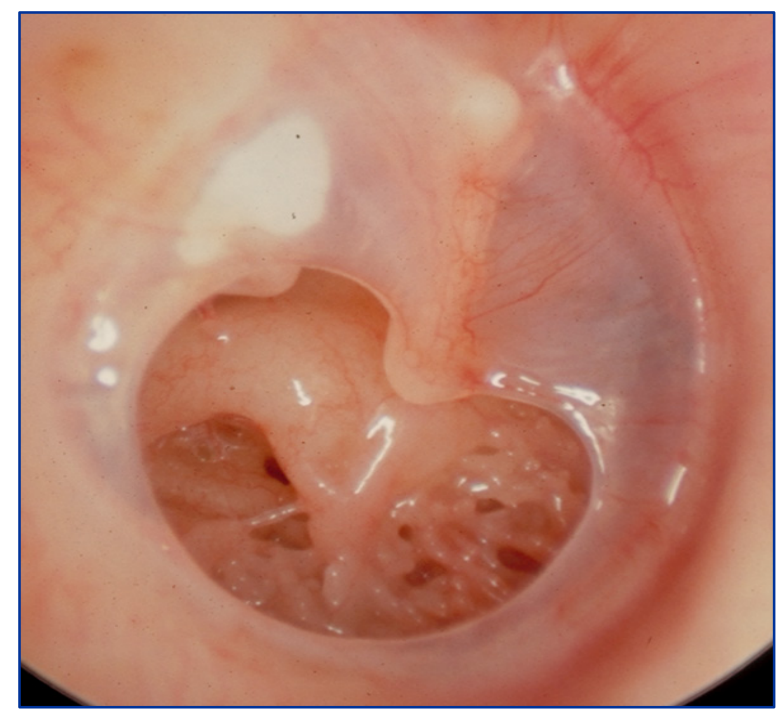

Figure 2. Right ear. Large perforation of the lower part of the eardrum, with a myringosclerosis of the residual posterior part. No otorrhea and no ossicular erosion. 


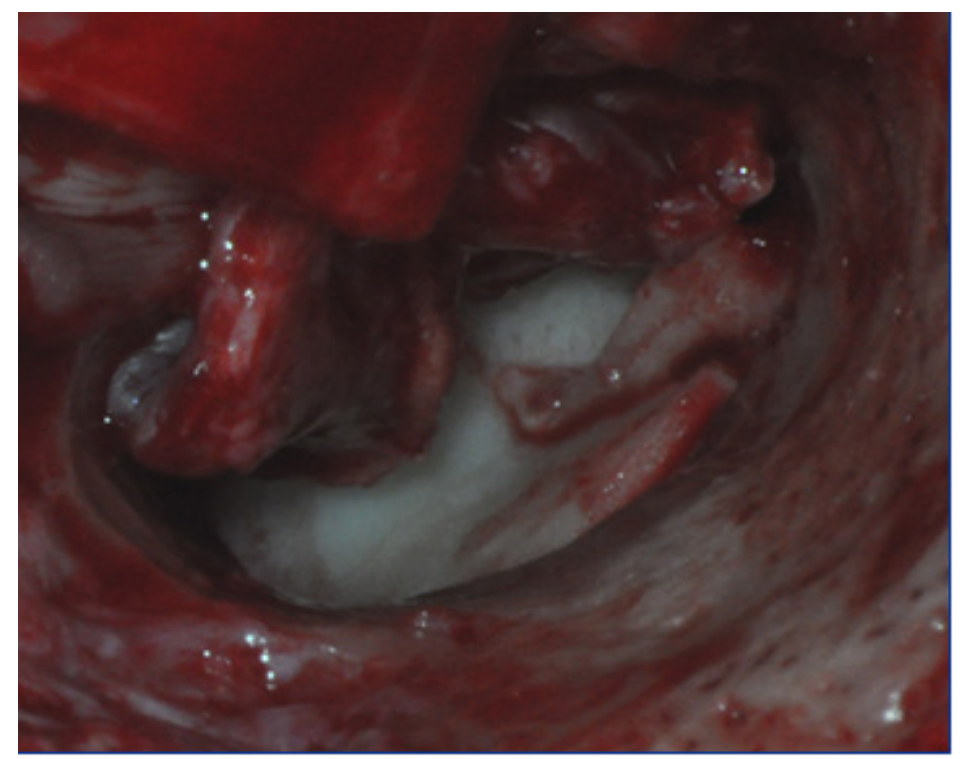

Figure 3. Intraoperative positioning under the malleus of a single tragal cartilage disk. We can see the calibrated auditory external canal and the elevated tympano-meatal flap.

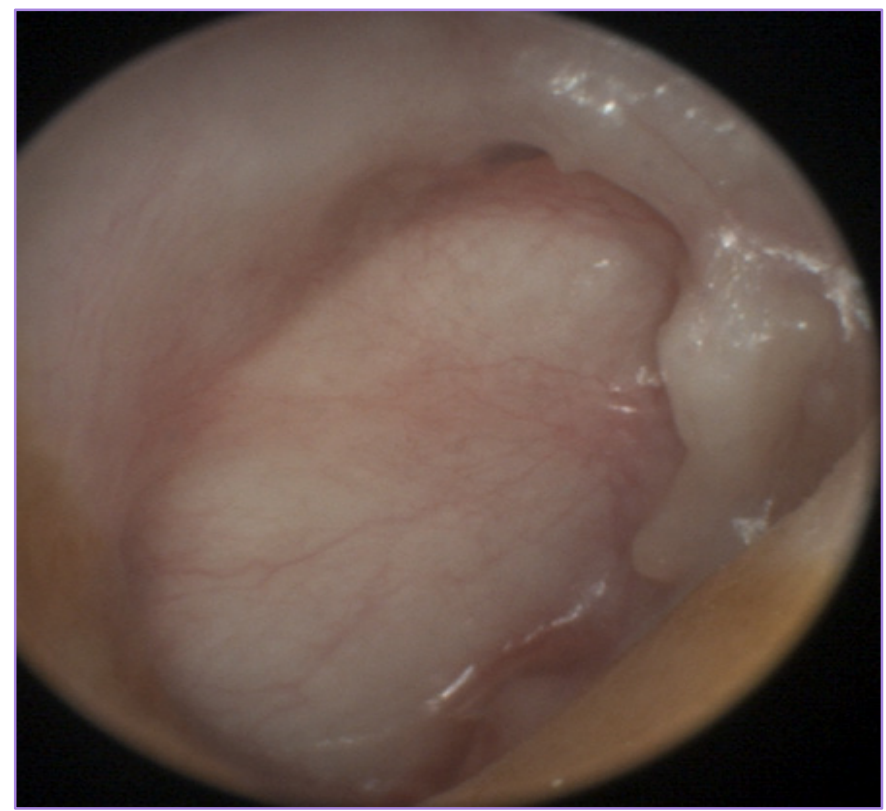

Figure 4. Eardrum after reconstruction with a single cartilage tragal disk. In this way we obtain a complete support to temporalis fascia, but we loose the transparency and the ability to monitor the middle ear.

\section{Results}

In our series of 63 cases, we had a success closure rate of 59/63 cases (94\%) with a medium follow-up of 36 months.

In the first 10 patients we noticed 4 cases of reperforation during the follow-up, always localized in the antero-superior portion of the eardrum, strictly near to cartilage graft. In fact during our first myringoplasties we were worried about a worse hearing result putting a complete cartilage graft under the residual tympanic membrane. So we were used to perform a cartilage reconstruction with a palisade technique. 
After this high rate of failure, we decided to place a single larger cartilaginous disk plus pericondrium, with the same size as the calibrated external auditory canal, in order to have a full support to temporalis fascia, avoiding the risk of future re-perforations. With this technical change we had a success rate of $100 \%$. This method may bring to a higher thickness of the eardrum that could increase the impedance of the transmission system. On the contrary, the Air Bone Gap (ABG) closure was always found from good to very good, going from a medium of 25-30 dB before surgery to 0-15 dB evaluated with an audiometric exam 6 months after surgery [Tab 1].

Table 1: Results considering Air-Bone Gap (ABG)

\begin{tabular}{llll}
\hline & Palizade Tech & Circle Tech & Tot Pts \\
\hline $\mathrm{ABG}<5 \mathrm{~dB}$ & 8 & 41 & 54 \\
$5 \mathrm{~dB}<\mathrm{ABG}<15 \mathrm{~dB}$ & 2 & 12 & 9 \\
$\mathrm{ABG}>20 \mathrm{~dB}$ & 0 & 0 & 0 \\
& 10 & 53 & 63 \\
\hline
\end{tabular}

We divided Pts in two groups, considering our first technique with palisade cartilage reconstruction and the final technique with a single tragal cartilage disk.

\section{Discussion}

\section{What should be the right moment to perform a myringoplasty?}

The Authors chose to operate children older than 5 years. We kept this decision for anatomical size of the outer ear. But it is mandatory to leave enough time to be sure of the behavior of the pathology: presence of granulation tissue, not-well defined margins, and polyps occupying the external auditory canal must be solved before planning surgery.

Vrabec et Al. thought that age is the only factor influencing a surgical successful outcome [3]. In Koch's opinion myringoplasty should be done in children over 8 years [4]; on the contrary, in some other papers, age is not cited as a determinant factor of success rate of pediatric tympanoplasty [5-6]. In these just cited articles, Yung starts surgery in children aged 4 and Albera starts from 3 years old patients.

\section{Which preoperative evaluations should be done?}

Bilateral otomicroscopy is the first procedure to evaluate the dryness, the site and the size of the perforation, the condition of the middle ear mucosa through the perforation. Contralateral ear must be checked to see potential bilateral perforation and retraction pockets.

We think that a complete audiological test is really important. We usually perform tonal audiometry and contralateral tympanometry. When feasible, we do a nasal endoscopy to evaluate adenoids size and finally a CT scan only in selected cases, if there is a suspicion of an epidermization of the tympanic cavity (chronic cholesteatomatous otitis) or a discontinuity of the ossicular chain.

\section{Healthy Eustachian tube.}

Hardman et Al. statistically analyzed Eustachian tube function, using tympanometry. They found no correlation between normal and abnormal function. Merenda et Al. [7] wrote that contralateral disease was associated with lower success rates. Conversely, Vartiainen et Al [8] and Pignataro et Al [9] found no correlation between contralateral ear status and surgical outcome. In this study we consider only monolateral tympanic perforation, with contralateral normal ear and normal Eustachian tube function. Therefore we can not give any indications about it, but we believe that a malfunctioning Eustachian tube is one of the main causes of failure for myringoplasty.

\section{What is the safest surgical technique that should be performed?}

The nature of the graft used to close perforation can be temporalis fascia and a tragal cartilage graft. 
We would like to underline that a combined underlay reconstruction may obtain always an excellent success rate. Underlay technique has the advantages of a rapid healing, rare complications like blunting or lateralization of the eardrum and a high graft take rate.

Disadvantages about this kind of surgery are a limited visualization, difficulty to treat large and/or anterior perforations, and problems with small external auditory canals. We go further these obstacles by using routinely canalplasty and a retroauricular approach.

\section{An endoscopic operating technique can offer comparable or better functional and anatomical results than traditional surgery?}

We don't have enough experience in endoscopic surgery but we think that a wider view due to the use of endoscope can be overcome by an adequate canalplasty that we usually perform. However in Literature endoscopic approaches seem to have higher success rates than microscopic ones [10-11-12].

\section{How much cartilage should we use?}

In the first 10 cases we used a reconstruction based on a palisade graft technique to support the temporalis fascia.

Due to our initial high incidence of reperforation (40\%), we modified our technique, using a single tragal cartilage disk with the same size as the calibrated external auditory canal, to sustain temporalis fascia. In this way we had a higher success rate (100\% against $60 \%)$ with very good hearing results. ABG closure was always 0-15 dB after 6 months of follow-up [Tab 1].

\section{Success rate}

There are many open questions regarding pediatric otologic surgery. We would like to discuss some of them regarding type I tympanoplasty, in the light of our fifteen years experience. About success rate we had $93,65 \%$ of closed myringoplasty after a follow-up of at least 18 months (mean period of surveillance of 36 months).

The Literature is mixed about that: in adults the success rate may vary from 60 to $99 \%$ but in the pediatric population it goes from 35 to $94 \%$ [13-14-15-16-17]. A possible variability could derive from the fact that ABG closure can be considered or not in the results.

The site and the size of the perforation are usually examined. In an article by Albera et Al. [6], after revision of 212 cases of myringoplasty (not only pediatric population), the Authors found some differences in the surgical outcome giving the site of the perforation: posterior perforations were found to have a higher incidence of failure than the anterior ones. In that article they often performed a transcanalar approach to close posterior perforations. In a publication by Pignataro et Al [9], after a revision of 41 pediatric patients, no difference about the surgical outcome was recorded putting in comparison anterior and posterior perforations.

In a very large clinical records by Lee [18], with an examination of 423 cases, and analysing the 607 patients by Emir [19] the results showed that the size of the perforation influence the outcome: smaller perforations ( $<50 \%$ of the tympanic membrane) have better chances to close than larger ones. Otherwise, a revision of 231 cases by Denoyelle [20] showed no difference between the success rate considering perforations of less than 25-between 25 and $50 \%$ and over $50 \%$ of the tympanic membrane.

In our cases we had no difference in success rate considering the site and the size of the tympanic perforations. We are strongly convinced that an adequate calibration of the external auditory canal has a key role to guarantee a stable reconstruction, even if in case of anterior or marginal perforations.

In our patients we mostly chose a retroauricular approach and the transcanalar one was an exception, performed in case of small posterior perforations and in case of particular conditions due to the patient (the older ones).

Finally we want to underline that the site of the re-perforation in our clinical records was noticed always at the margin of the cartilage grafts. So we modified our technique, using a single tragal cartilage disk with the same size as the calibrated external auditory canal, to sustain temporalis fascia. In this way we passed from a success rate of $60 \%$ to a success rate of $100 \%$, always with an ABG closure within 15 dB 6 months after surgery. 


\section{Limits of our study}

This study has some limits: in particular a small number of cases and a brief median period of surveillance (36 months). This can be good to evaluate stability of hearing results but we don't think it can be enough to exclude the occurrence of iatrogenic cholesteatoma.

Furthermore, our study population is characterized by a normal contralateral ear. In our opinion this feature is a guarantee for a good functional and stable result. Obviously it is common experience that we can't often find this feature, because the first cause of tympanic perforation in children is the bilateral tubaric dysfunction.

\section{Conclusions}

Myringoplasty is a safe surgical procedure that helps pediatric patients to return to a good hearing level, to reduce the frequency of infections and to allow them to practice water sports. It can be performed in addition to adenoidectomy-adenotonsillectomy.

The minimum age to perform this surgery is 5 years, in our experience.

In our series of 63 cases we had an overall success rate of $94 \%$, considering no preoperative infections and a good contralateral ear function.

\section{Conflicts of interest: none}

This research did not receive any specific grant from funding agencies in the public, commercial, or notfor-profit sectors.

\section{References}

1. Tos M, Lau T. Stability of tympanoplasty in children. Otolaryngol Clin North Am. 1989; 22:15-28

2. Harman J, Muzaffar J, Nankivell P and Coulson C. Tympanoplasty for chronic tympanic membrane perforation in children: systematic review and meta-analysis. Otology \& neurotology 2015; 36: 796-804

3. Vrabec JT, Deskin RW, Grady JJ. Meta-analysis of paediatric tympanoplasty. Arch Otolaryngol Head Neck Surg. 1999;125:530-534

4. Koch WM, Friedman EM. McGill TJ, Healy GB. Tympanoplasty in children. The Boston Children's Hospital experience. Arch Otolaryngol Head Neck Surg. 1990; 116:35-40

5. Young M, Newman C, Vowler SL. A longitudinal study on paediatric tympanoplasty. Otol Neurotol. 2007; 28:353355

6. Albera R, Ferrero V, Lacilla M, Canale A. Tympanic perforation in miringoplasty: evaluation of prognostic factors. Ann Otol Laryngol . 2006; 115:875-879

7. Merenda D, Koike K, Shafiei M, Ramadan H. Tympanometric volume: a predictor of success of tympanoplasty in children. Otolaryngol Head neck Surg.2007;136:189-192

8. Vartiainen E, Vartiainen J. Tympanoplasty in young patients: the role of adenoidectomy. Otolaryngol Head Neck Surg. 1997 Dec;117(6):583-5.

9. Pignataro L, Grillo Della Berta L, Capaccio P, Zaghis A. Myringoplasty in children: anatomical and functional results. J Laryngol Otol. 2001 May;115(5):369-73.

10. Nassif N, Berlucchi M, Redaelli De Zinis LO. Tympanic membrane perforation in children: endoscopic type I tympanoplasty, a newly technique, is it worthwhile? Int J Pediatr Otorhinolaryngol. 2015 Nov; 79(11):1860-4

11. Migirov L, Wolf M. Transcanal microscope-assisted endoscopic myryngoplasty in children. BMC pediatr. 2015 Apr $1 ; 15-32$

12. Akyigit A, Karlidag T, Keles E et Al. Endoscopic cartilage butterfly myringoplasty in children. Auris Nasus Larynx. 2016 Jun 1

13. Umapathy N, Dekker PJ. Myringoplasty: is it worth performing in children? Arch Otolaryngol Head Neck Surg. 2003 Oct;129(10):1053-5.

14. Halim A, Borgstein J. Pediatric myringoplasty: postaural versus transmeatal approach. Int J Pediatr Otorhinolaryngol. 2009 Nov;73(11):1580-3. doi: 10.1016/j.ijporl.2009.08.010. Epub 2009 Sep 6.

15. Castelli ML, Vitiello R, Ponzo S, Evangelista A. Ten years' experience with Felix tympanoplasty: analysis of 
anatomical and functional results. J Laryngol Otol. 2015 Nov;129(11):1064-8. doi: 10.1017/S0022215115002297. Epub 2015 Sep 22.

16. Abdellatif H, Youssef R, Omar M, Hassan N, Lahcen A, Abdelaziz R. Myringoplasty in children: retrospective analysis of 60 cases. Pan Afr Med J. 2015 Jan 29;20:82. doi: 10.11604/pamj.2015.20.82.3620. eCollection 2015

17. Al-Khtoum N, Hiari MA. Myringoplasty in children: retrospective analysis of 35 cases. Braz J Otorhinolaryngol. 2009 May-Jun;75(3):371-4. English, Portuguese.

18. Lee P, Kelly G, Mills RP. Myringoplasty: does the size of the perforation matter? Clin Otolaryngol Allied Sci. 2002 Oct;27(5):331-4.

19. Emir H, Ceylan K, Kizilkaya Z, Gocmen H, Uzunkulaoglu H, Samim E. Success is a matter of experience: type 1 tympanoplasty : influencing factors on type 1 tympanoplasty. Eur Arch Otorhinolaryngol. 2007 Jun;264(6):595-9. Epub 2007 Jan 18.

20. Denoyelle F, Roger G, Chauvin P, Garabedian EN. Myringoplasty in children. Predictive factors of outcome. Laryngoscope 1999 Jan; 109(1):47-51 